\title{
On the coordinate functions of Lévy's dragon curve
}

\author{
Pieter C. Allaart and Kiko Kawamura \\ University of North Texas *
}

February 9, 2006

\begin{abstract}
Lévy's dragon curve [P. LÉvy, Les courbes planes ou gauches et les surfaces composées de parties semblables au tout, J. Ecole Polytechn., 227-247, 249-291 (1938)] is a well-known self-similar planar curve with non-empty interior. We derive an arithmetic expression for the coordinate functions of Lévy's dragon curve, and show that the $\frac{3}{2}$-dimensional Hausdorff measure of the graph of each coordinate function is strictly positive and finite. This complements known dimensional results concerning the coordinate functions of space-filling curves of Peano and Hilbert. The proof is based on deriving suitable uniform upper bounds for the sizes of the graphs' level sets.
\end{abstract}

\section{Introduction}

Lévy's dragon curve $\mathcal{D}$ (Figure 1) was introduced and studied in 1938 by P. Lévy [13]. It is the unique compact set in the complex plane satisfying the set equation

$$
\mathcal{D}=\psi_{1}(\mathcal{D}) \cup \psi_{2}(\mathcal{D}),
$$

where $\psi_{1}$ and $\psi_{2}$ are similar contractions given by $\psi_{1}(z)=\left(\frac{1}{2}+\frac{1}{2} i\right) z$, and $\psi_{2}(z)=\left(\frac{1}{2}-\frac{1}{2} i\right) z+\left(\frac{1}{2}+\frac{1}{2} i\right)$. Figure 2 shows how $\mathcal{D}$ can be constructed from the isosceles triangle $A_{0}$ with vertices 0,1 , and $\frac{1}{2}+\frac{1}{2} i$. It is easy to see from this recursive construction that the area of $\mathcal{D}$ is the same as that of $A_{0}$. In fact, it is known that $\mathcal{D}$ has non-empty interior, and can be used to tile the complex plane. Other known results on Lévy's dragon curve concern the Hausdorff dimension of its boundary [3], and a classification of the regions "inside" Lévy's dragon (the connected components of $\mathcal{D}^{c}$ ) [1]. An English translation of Lévy's original paper is included in Edgar's book [4].

*Mathematics Department, P.O. Box 311430, Denton, TX 76203-1430; e-mail: allaart@unt.edu, kiko@unt.edu

AMS 2000 subject classification: Primary 28A78, 28A80; Secondary 26A27, 26A30.

Key words and phrases: Lévy's dragon curve, coordinate functions, Hausdorff dimension, self-affine function 


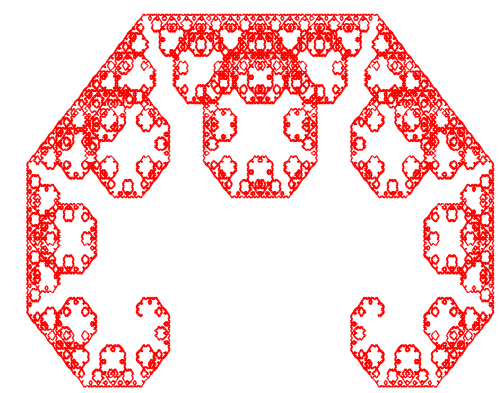

Figure 1: Lévy's dragon curve

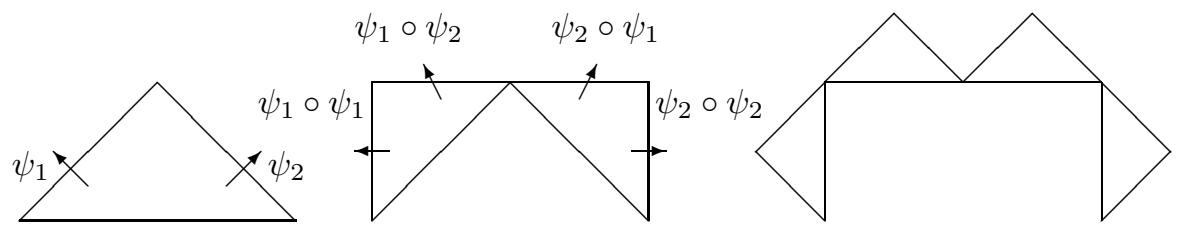

$A_{0}$

$A_{1}$

$A_{2}$

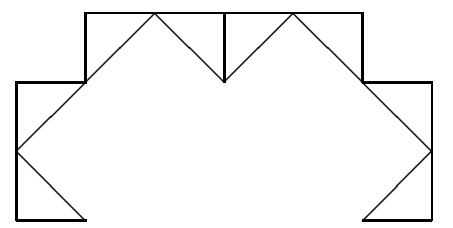

$A_{3}$

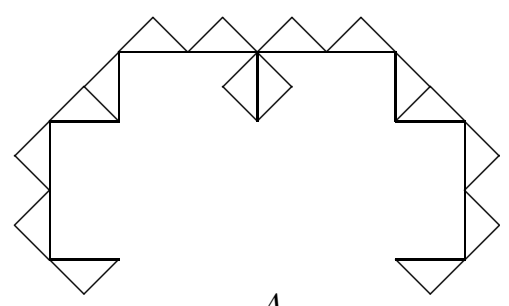

$A_{4}$

Figure 2: The first five steps of the construction of Lévy's dragon curve

Equation (1) yields the natural parametrization $\{L(t): 0 \leq t \leq 1\}$ of $\mathcal{D}$, where $L(t)$ is the unique continuous solution of the functional equation

$$
L(t)= \begin{cases}\left(\frac{1}{2}+\frac{1}{2} i\right) L(2 t), & 0 \leq t \leq \frac{1}{2}, \\ \left(\frac{1}{2}-\frac{1}{2} i\right) L(2 t-1)+\left(\frac{1}{2}+\frac{1}{2} i\right), & \frac{1}{2} \leq t \leq 1 .\end{cases}
$$

Write $L(t)=x(t)+i y(t)$. We will be interested in the coordinate functions $x(t)$ and $y(t)$.

The functional equation (2) belongs to a class of functional equations studied by G. de Rham in 1957 [2], namely

$$
G_{\alpha}(t)= \begin{cases}\alpha G_{\alpha}(2 t), & 0 \leq t \leq \frac{1}{2}, \\ (1-\alpha) G_{\alpha}(2 t-1)+\alpha, & \frac{1}{2} \leq t \leq 1\end{cases}
$$


where $\alpha$ is a complex parameter satisfying $|\alpha|<1,|1-\alpha|<1$. (See also page 288 of [5]. The functional equation is stated here in parametrized form to facilitate comparison with (2).)

De Rham showed that (3) has a unique continuous solution $G_{\alpha}$, and that the real and imaginary parts of $G_{\alpha}(t)$ are nowhere differentiable when $\min \{|\alpha|, \mid 1-$ $\alpha \mid\}>1 / 2$. Since in the case of $L(t)$ we have $\alpha=\frac{1}{2}+\frac{1}{2} i$, it follows that $x(t)$ and $y(t)$ are nowhere-differentiable.

Next, since $\mathcal{D}$ is self-similar, we expect $x(t)$ and $y(t)$ to be self-affine in some sense. It is of particular interest to ask for the Hausdorff dimension of their graphs, which we denote by $X$ and $Y$, respectively. While there is a wealth of literature on the Hausdorff dimensions of self-affine sets, it seems that none of the general results is directly (and easily) applicable to this specific case. Instead, we give an argument "from the ground up", using little more than the definition of Hausdorff measure and by showing that the level sets of the graphs do not become "too large", to prove that $X$ and $Y$ have positive and finite $\frac{3}{2}$-dimensional Hausdorff measure. This complements known results for the coordinate functions of space-filling curves of Peano [12] and Hilbert [15].

The key to viewing the coordinate functions is to iterate the functional equation (2), obtaining

$$
\left(x\left(\frac{k+t}{4}\right), y\left(\frac{k+t}{4}\right)\right)= \begin{cases}(0,0)+\left(\frac{-y(t)}{2}, \frac{x(t)}{2}\right), & \text { if } k=0, \\ \left(0, \frac{1}{2}\right)+\left(\frac{x(t)}{2}, \frac{y(t)}{2}\right), & \text { if } k=1, \\ \left(\frac{1}{2}, \frac{1}{2}\right)+\left(\frac{x(t)}{2}, \frac{y(t)}{2}\right), & \text { if } k=2, \\ \left(1, \frac{1}{2}\right)+\left(\frac{y(t)}{2}, \frac{-x(t)}{2}\right), & \text { if } k=3,\end{cases}
$$

for $0 \leq t \leq 1$. The boundary conditions are $x(0)=0, x(1)=1$ and $y(0)=$ $y(1)=0$. This functional equation closely resembles the functional equation for the coordinate functions of Hilbert's space filling curve (see [15]). The only difference is, that the signs of $y(t)$ in the cases $k=0$ and $k=3$ are inverted. However, this difference makes the study of level sets, which were used successfully by McClure [15] to obtain the dimension $\frac{3}{2}$ for Hilbert's coordinate functions, much more delicate in our case.

From (4), we see that $X$ and $Y$ each consist of two affine contracted images of $X$ and two of $Y$. (See Figure 3). Thus, the functions $x(t)$ and $y(t)$ are selfaffine in the sense of Kamae [10], but not in the sense of Kono [12]. Moreover, since the contraction ratio in the vertical direction $(1 / 2)$ is different from that in the horizontal direction $(1 / 4)$, standard dimension theorems of Hutchinson [9] and Mauldin and Williams [14] do not apply here.

When the affine images of $X$ and $Y$ are mixed together as in Figure 3, it is usually more difficult to obtain the Hausdorff dimensions of $X$ and $Y$. Kenyon and Peres [11] and Takahashi [17] give general formulas for the dimensions of self-affine sets determined by multiple patterns. However, these formulas require the affine contracted images of the patterns to be aligned in horizontal strips. While the graphs of Hilbert's coordinate functions satisfy this property (see 


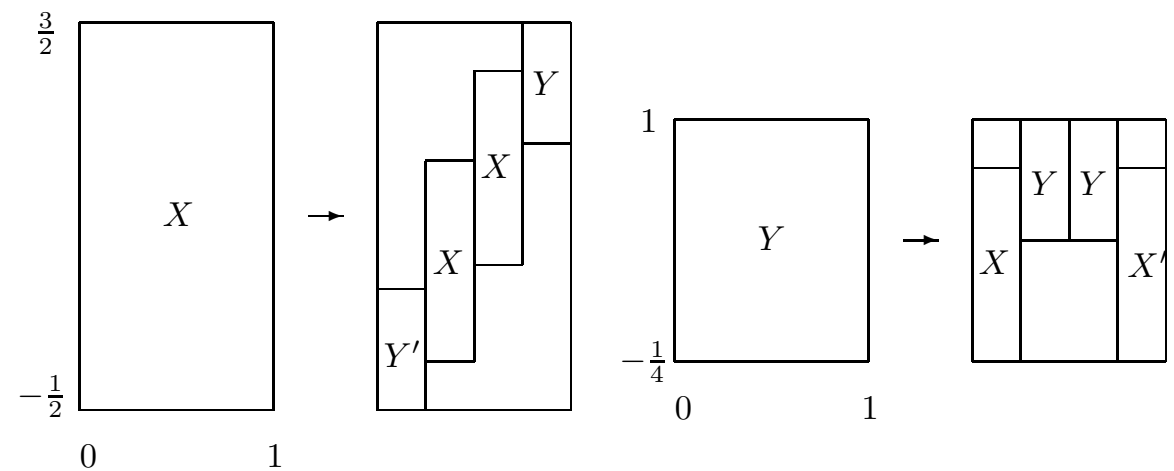

Figure 3: The structure of $X$ and $Y$. Prime symbols (') indicate a top-to-bottom reflection of the affine image.

[15]), it clearly fails for the graphs of Lévy's coordinate functions. Precisely, the projections of the affine images of $X$ and $Y$ onto the vertical axis partially overlap each other. While Kenyon and Peres [11] indicate a general method to reduce this "overlap" case to the aligned case, the calculations involved can be quite overwhelming, and even if the correct dimension is obtained, there is no guarantee that $X$ and $Y$ will have positive and finite Hausdorff measure with respect to this dimension. In many individual cases it is more practical to look for a direct argument (often involving level sets), such as that used by Edgar [5] for Kiesswetter's fractal. This is the approach taken in the present article.

The details concerning the Hausdorff dimension of $X$ and $Y$ are given in section 3. But first, in section 2, we adapt Sagan's method in [16] to give arithmetic expressions for $x(t)$ and $y(t)$ in terms of the quaternary expansion of $t$. While not needed to obtain the Hausdorff dimension, these expressions are included here since they are useful for producing graphs of $x(t)$ and $y(t)$, and they do not seem to have appeared in print before.

\section{Arithmetization of Lévy's coordinate functions}

Since the curve $L(t)$ is defined by two complex contractions, it seems natural to give a representation of $L(t)$ using the binary expansion of $t$. Indeed, such an expression was given by Lévy in his 1938 paper. A disadvantage of this approach is that it is not clear how to distill elementary expressions for the coordinates from this complex representation.

To overcome this problem, we consider the second iterates of the mappings $\psi_{1}$ and $\psi_{2}$ defined in the introduction: let $\mathcal{L}_{0}=\psi_{1} \circ \psi_{1}, \mathcal{L}_{1}=\psi_{1} \circ \psi_{2}, \mathcal{L}_{2}=\psi_{2} \circ \psi_{1}$, and $\mathcal{L}_{3}=\psi_{2} \circ \psi_{2}$. Each $\mathcal{L}_{q}$ maps the closed rectangle $G$ with vertices $-1 / 2-i / 4$, $-1 / 2+i, 3 / 2-i / 4$ and $3 / 2+i$ into itself. 
We now follow Sagan's method in [16]. For $0 \leq t \leq 1$, let

$$
t=0 . q_{1} q_{2} q_{3} \ldots
$$

denote the quaternary expansion of $t$. (In case $t$ has two different quaternary expansions, either representation may be chosen.) Then for each $n$,

$$
L(t) \in \mathcal{L}_{q_{1}} \circ \mathcal{L}_{q_{2}} \circ \cdots \circ \mathcal{L}_{q_{n}}(G),
$$

and since the images of $G$ under $\mathcal{L}_{q_{1}} \circ \mathcal{L}_{q_{2}} \circ \cdots \circ \mathcal{L}_{q_{n}}$ shrink to a point as $n \rightarrow \infty$,

$$
\{L(t)\}=\lim _{n \rightarrow \infty} \mathcal{L}_{q_{1}} \circ \mathcal{L}_{q_{2}} \circ \cdots \circ \mathcal{L}_{q_{n}}(G)
$$

(where the convergence is with respect to the Hausdorff distance between sets). Clearly, we may replace $G$ in the above limit with the single point $0 \in G$, and write

$$
L(t)=\lim _{n \rightarrow \infty} \mathcal{L}_{q_{1}} \circ \mathcal{L}_{q_{2}} \circ \cdots \circ \mathcal{L}_{q_{n}}(0) .
$$

Now the transformations $\mathcal{L}_{q}$ may be written in the form

$$
\mathcal{L}_{q} z=\frac{1}{2} L_{q} z+\frac{1}{2} l_{q}, \quad q=0,1,2,3,
$$

where $L_{0} z=z i, L_{1} z=L_{2} z=z, L_{3} z=-z i, l_{0}=0$, and $l_{q}=q-1+i$ for $q=1,2,3$. By successive substitution of (6) into (5), we obtain that

$$
\mathcal{L}_{q_{1}} \circ \mathcal{L}_{q_{2}} \circ \ldots \circ \mathcal{L}_{q_{n}}(0)=\sum_{j=1}^{n}\left(\frac{1}{2^{j}}\right) L_{q_{1}} L_{q_{2}} \ldots L_{q_{j-1}} l_{q_{j}},
$$

where $L_{q_{1}} L_{q_{2}} \ldots L_{q_{j-1}}$ is interpreted as the identity if $j=1$. Now let $n_{0, j}$ and $n_{3, j}$ denote respectively the number of 0's and the number of 3's among $q_{1}, \ldots, q_{j-1}$, and define

$$
d_{j}:=n_{0, j}-n_{3, j} \quad(\bmod 4) .
$$

Observe that

$$
L_{q_{1}} L_{q_{2}} \ldots L_{q_{j-1}}=L_{0}^{d_{j}},
$$

and so,

$$
L(t)=\lim _{n \rightarrow \infty} \sum_{j=1}^{n}\left(\frac{1}{2^{j}}\right) L_{0}^{d_{j}} l_{q_{j}}=\sum_{j=1}^{\infty}\left(\frac{1}{2^{j}}\right) i^{d_{j}} l_{q_{j}} .
$$

Writing $l_{q_{j}}=\operatorname{sgn}\left(q_{j}\right)\left(q_{j}-1+i\right)$, and considering each of the four possible values of $d_{j}$ separately, we obtain the following expressions for the real and imaginary parts of $L(t)$ :

$$
\left(\begin{array}{l}
x(t) \\
y(t)
\end{array}\right)=\sum_{j=1}^{\infty}\left(\frac{1}{2^{j}}\right)\left(\begin{array}{c}
(-1)^{\left\lfloor d_{j} / 2\right\rfloor} \\
(-1)^{\left\lceil d_{j} / 2\right\rceil}
\end{array}\right) \operatorname{sgn}\left(q_{j}\right)\left(\begin{array}{c}
\left(1-d_{j} \bmod 2\right) q_{j}-1 \\
1-\left(d_{j} \bmod 2\right) q_{j}
\end{array}\right),
$$


where $\lfloor x\rfloor$ and $\lceil x\rceil$ denote the largest integer less than or equal to $x$ and the smallest integer greater than or equal to $x$, respectively. Notice how this expression utilizes both the quotient and the remainder of $d_{j}$ divided by 2 .

It remains to be checked that for points $t$ with a terminating quaternary expansion, the expression (8) does not depend on the representation chosen:

$$
t=0 . q_{1} \ldots q_{n-1} q, \quad \text { or } \quad t=0 . q_{1} \ldots q_{n-1}(q-1) \overline{3} .
$$

Let $d_{n+1}^{\prime}=d_{n}+1_{\{q=1\}}$. Then

$$
L\left(0 . q_{1} \ldots q_{n-1} q\right)=\sum_{j=1}^{n-1}\left(\frac{1}{2^{j}}\right) i^{d_{j}} l_{q_{j}}+\left(\frac{1}{2^{n}}\right) i^{d_{n}} l_{q},
$$

and

$$
\begin{aligned}
& L\left(0 . q_{1} \ldots q_{n-1}(q-1) \overline{3}\right)=\sum_{j=1}^{n-1}\left(\frac{1}{2^{j}}\right) i^{d_{j}} l_{q_{j}}+\left(\frac{1}{2^{n}}\right) i^{d_{n}} l_{q-1} \\
& +\left(\frac{1}{2^{n+1}}\right) i^{d_{n+1}^{\prime}}\left[1-\frac{i}{2}+\left(\frac{i}{2}\right)^{2}-\left(\frac{i}{2}\right)^{3}+\ldots\right](i+2) .
\end{aligned}
$$

Noting that

$$
\left[1-\frac{i}{2}+\left(\frac{i}{2}\right)^{2}-\left(\frac{i}{2}\right)^{3}+\ldots\right](i+2)=2,
$$

we obtain that

$$
\begin{aligned}
L\left(0 . q_{1} \ldots q_{n-1}(q-1) \overline{3}\right)- & L\left(0 . q_{1} \ldots q_{n-1} q\right) \\
= & \left(\frac{1}{2^{n}}\right)\left[i^{d_{n}}\left(l_{q-1}-l_{q}\right)+i^{d_{n+1}^{\prime}}\right] \\
= & \left(\frac{1}{2^{n}}\right) i^{d_{n}}\left[l_{q-1}-l_{q}+1+(i-1) 1_{\{q=1\}}\right] \\
= & 0
\end{aligned}
$$

where the last equality follows by recalling that $l_{0}=0$, and $l_{q}=q-1+i$ for $q \geq 1$.

Expression (9) allows us to plot accurate approximations of the graphs of $x(t)$ and $y(t)$, as shown in Figure 4. Note that the graphs are scaled differently. These graphs may be compared to the symbolic representations in Figure 3.

\section{The Hausdorff dimension of the coordinate functions}

We first recall the definition of Hausdorff measure and Hausdorff dimension below. For a set $F$ in Euclidean space, let $|F|$ denote the diameter of $F$. Let 

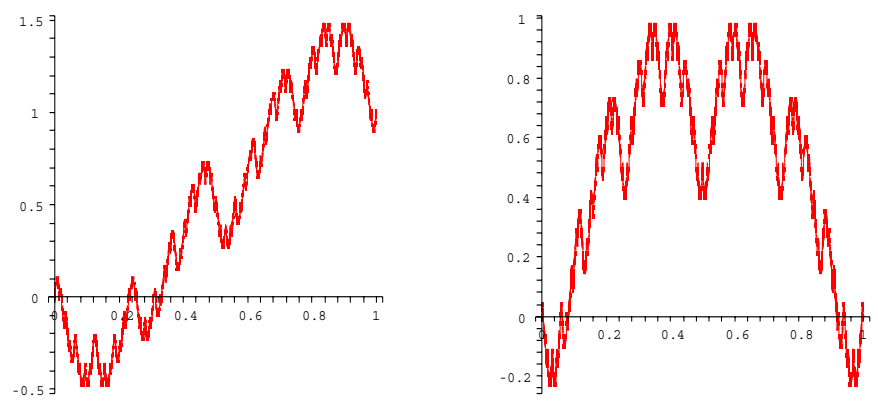

Figure 4: Graphs of $x(t)$, left, and $y(t)$, right.

$s \geq 0$. For $F \in \mathbf{R}^{n}$ and $\delta>0$, define

$$
\mathcal{H}_{\delta}^{s}(F):=\inf \left\{\sum_{i \in I}\left|U_{i}\right|^{s}: F \subseteq \bigcup_{i \in I} U_{i} \quad \text { and } \quad\left|U_{i}\right|<\delta \quad \text { for every } i \in I\right\},
$$

where $I$ denotes a countable index set. The $s$-dimensional Hausdorff measure of $F$ is defined by

$$
\mathcal{H}^{s}(F):=\lim _{\delta \rightarrow 0^{+}} \mathcal{H}_{\delta}^{s}(F),
$$

and the Hausdorff dimension of $F$ is defined by

$$
\operatorname{dim} F:=\sup \left\{s \geq 0: \mathcal{H}^{s}(F)=\infty\right\}=\inf \left\{s \geq 0: \mathcal{H}^{s}(F)=0\right\} .
$$

For a broader introduction to Hausdorff dimension, see Falconer [6].

Recall that $X$ is the graph of $x(t)$, and $Y$ is the graph of $y(t)$. More precisely, $X:=\{(t, x(t)): 0 \leq t \leq 1\}$ and $Y:=\{(t, y(t)): 0 \leq t \leq 1\}$. Our purpose is to prove the following theorem.

Theorem 3.1 (i) $\operatorname{dim} X=\operatorname{dim} Y=\frac{3}{2}$; and (ii) $0<\mathcal{H}^{3 / 2}(X)=\mathcal{H}^{3 / 2}(Y)<\infty$.

The proof will follow from the observations and lemmas below. Note first that, using (4) and the continuity of $x(t)$ and $y(t)$, it is straightforward to show that

$$
x(t)=1-x(1-t), \quad \text { and } \quad y(t)=y(1-t), \quad 0 \leq t \leq 1 .
$$

Let $Z$ denote the left half of $X$. That is, $Z:=\{(t, x(t)): 0 \leq t \leq 1 / 2\}$. In view of the above symmetries, $X$ consists of two congruent copies of $Z$ (the second being rotated $180^{\circ}$ ). Similarly, $Y$ consists of two congruent copies of $Z$ (the first one rotated $180^{\circ}$, and the second reflected top-to-bottom). This can be seen from (4), which shows that the left half of $Y$ consists of an affine-contracted 
copy of $X$ followed by an affine-contracted copy of $Y$ itself (each reduced horizontally by a factor 4 and vertically by a factor 2). By the symmetries (10), this combination is congruent to $Z$.

It follows from the above reasoning that $Z$ consists of four affine-contracted copies of itself, and that $\operatorname{dim} X=\operatorname{dim} Y=\operatorname{dim} Z$. Thus, we have reduced the sets of interest $(X$ and $Y$ ) to essentially one single self-affine set $(Z)$.

It is slightly more convenient to consider the set $\hat{Z}:=2 Z:=\{(2 t, 2 z)$ : $(t, z) \in Z\}$. From the above facts, $\hat{Z}$ is the unique nonempty compact set satisfying the set equation

$$
\hat{Z}=\bigcup_{i=1}^{4} T_{i}(\hat{Z}),
$$

where

$$
\begin{aligned}
& T_{1}\left(\begin{array}{l}
t \\
z
\end{array}\right)=\left[\begin{array}{cc}
-1 / 4 & 0 \\
0 & 1 / 2
\end{array}\right]\left[\begin{array}{l}
t \\
z
\end{array}\right]+\left[\begin{array}{c}
1 / 4 \\
-1 / 2
\end{array}\right], \\
& T_{2}\left(\begin{array}{l}
t \\
z
\end{array}\right)=\left[\begin{array}{cc}
1 / 4 & 0 \\
0 & 1 / 2
\end{array}\right]\left[\begin{array}{l}
t \\
z
\end{array}\right]+\left[\begin{array}{c}
1 / 4 \\
-1 / 2
\end{array}\right], \\
& T_{3}\left(\begin{array}{l}
t \\
z
\end{array}\right)=\left[\begin{array}{cc}
1 / 4 & 0 \\
0 & 1 / 2
\end{array}\right]\left[\begin{array}{l}
t \\
z
\end{array}\right]+\left[\begin{array}{c}
1 / 2 \\
0
\end{array}\right] \\
& T_{4}\left(\begin{array}{l}
t \\
z
\end{array}\right)=\left[\begin{array}{cc}
-1 / 4 & 0 \\
0 & -1 / 2
\end{array}\right]\left[\begin{array}{l}
t \\
z
\end{array}\right]+\left[\begin{array}{l}
1 \\
1
\end{array}\right]
\end{aligned}
$$

(See Figure 5.) A formula of Falconer [7] can be applied to these contractions to show that $\operatorname{dim} \hat{Z}=3 / 2$ is "almost certainly" true in a measure theoretic sense, and that $3 / 2$ is certainly an upper bound. Moreover, another result by Falconer [8, Corollary 5] implies that the box-counting dimension of $\hat{Z}$ is $3 / 2$. However, we still need to prove that $\operatorname{dim} \hat{Z}=3 / 2$ is "certainly" true.

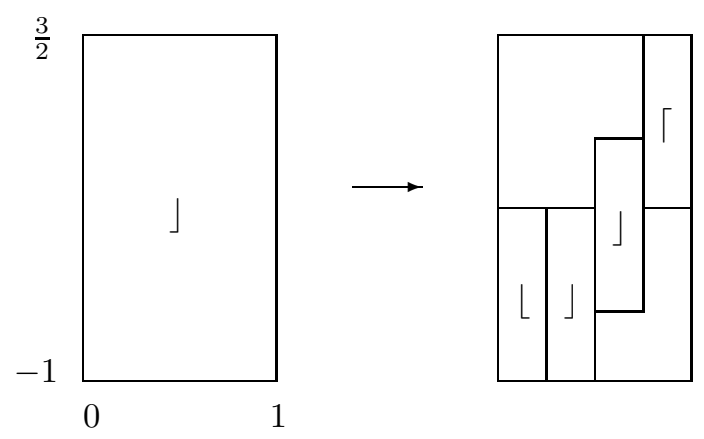

Figure 5: The self-affine structure of $\hat{Z}$

By considering the heights of the triangles in Figure 2, it is easily verified that the ranges of $x(t)$ and $y(t)$ are $\left[-\frac{1}{2}, \frac{3}{2}\right]$ and $\left[-\frac{1}{4}, 1\right]$, respectively. Thus, the 
smallest rectangle with sides parallel to the coordinate axes that contains $\hat{Z}$ is the rectangle $R:=[0,1] \times\left[-1, \frac{3}{2}\right]$. Now for $n \in \mathbf{N}$, define the stage- $n$ rectangles $R_{i_{1}, \ldots, i_{n}}$ by

$$
R_{i_{1}, \ldots, i_{n}}=T_{i_{1}} \cdots T_{i_{n}}(R), \quad 1 \leq i_{1}, \ldots, i_{n} \leq 4 .
$$

Figure 5 shows the stage- 1 rectangles with their orientations. Note that for all $n$,

$$
\hat{Z} \subseteq \bigcup_{i_{1}, \ldots, i_{n}} R_{i_{1}, \ldots, i_{n}}
$$

Lemma $3.2 \mathcal{H}^{3 / 2}(\hat{Z})<\infty$.

Proof. Each stage- $n$ rectangle has width $(1 / 4)^{n}$ and height $(5 / 2)(1 / 2)^{n}$, and can therefore be partitioned into $5 \cdot 2^{n-1}$ squares of side $(1 / 4)^{n}$. Since there are $4^{n}$ rectangles total at stage $n$, it follows from (11) that

$$
\mathcal{H}_{4^{-n} \sqrt{2}}^{3 / 2}(\hat{Z}) \leq 4^{n} \cdot 5 \cdot 2^{n-1}\left(4^{-n} \sqrt{2}\right)^{3 / 2}=5 \cdot 2^{-1 / 4},
$$

and the lemma follows upon letting $n \rightarrow \infty$.

The next lemma says roughly that the horizontal cross sections of $\hat{Z}$ do not become too large. It uses the following notation. For $z \in\left[-1, \frac{3}{2}\right]$ and $k=0,1,2, \ldots$, let

$$
\begin{gathered}
N_{k}(z)=\text { the number of stage- } k \text { rectangles intersected by } \\
\text { the line segment }[0,1] \times\{z\} .
\end{gathered}
$$

Define $N_{k}(z)=0$ if $z \notin\left[-1, \frac{3}{2}\right]$. Observe that for fixed $k, N_{k}(z)$ is constant on each interval $\left(j / 2^{k+1},(j+1) / 2^{k+1}\right)$, for $j \in \mathbf{Z}$.

Lemma 3.3 For each $z \in\left[-1, \frac{3}{2}\right]$ and $k \in \mathbf{N}$,

$$
N_{k}(z) \leq 6 \cdot 2^{k} .
$$

Proof. From the affine transformations that define $\hat{Z}$, we can derive the recursive relation

$$
N_{k}(z)=2 N_{k-1}(2 z+1)+N_{k-1}(2 z)+N_{k-1}(2-2 z), \quad-1 \leq z \leq \frac{3}{2}, \quad k \in \mathbf{N} .
$$

Define the mappings $f_{0}(z)=2 z+1, f_{1}(z)=2 z$, and $f_{2}(z)=2-2 z$. Let $I_{k}:=\{0,1,2\}^{k}$. For a sequence $\mathbf{i}=\left(i_{1}, \ldots, i_{k}\right)$ in $I_{k}$, define $|\mathbf{i}|:=k$, and let $f_{\mathbf{i}}$ denote the composition

$$
f_{\mathbf{i}}:=f_{i_{k}} \circ f_{i_{k-1}} \circ \cdots \circ f_{i_{1}} .
$$

Let $\mathcal{F}_{k}:=\left\{f_{\mathbf{i}}, \mathbf{i} \in I_{k}\right\}$. Note that each $f \in \mathcal{F}_{k}$ is of the form $f(z)=2^{k} z+j$ or $f(z)=-2^{k} z+j$, where $j \in \mathbf{Z}$. 
For $f \in \mathcal{F}_{k}$, let $\mathcal{P}(f)$ denote the set of predecessors of $f$. That is, $\mathcal{P}(f)$ is the set of all functions $\hat{f}$ in $\mathcal{F}_{k-1}$ such that $f=f_{i} \circ \hat{f}$ for some $i \in\{0,1,2\}$.

Now for each $z \in\left[-1, \frac{3}{2}\right]$, repeated iteration of (12) yields the representation

$$
N_{k}(z)=\sum_{f \in \mathcal{F}_{k}} c_{f}(z) N_{0}(f(z)) .
$$

Here the coefficients $c_{f}(z)$ are given by

$$
c_{f}(z)=\sum_{\mathbf{i}: f_{\mathbf{i}}=f} 2^{n(\mathbf{i})}
$$

where $n(\mathbf{i})=\operatorname{card}\left\{r: 1 \leq r \leq|\mathbf{i}|, i_{r}=0\right\}$, the number of zeros in $\mathbf{i}$.

Now observe that $N_{0}(f(z))=1$ if $f(z) \in\left[-1, \frac{3}{2}\right]$, and $=0$ otherwise. Since the greatest possible length of an arithmetic progression with spacing 1 that fits inside the interval $\left[-1, \frac{3}{2}\right]$ is 3 , it follows that at most 6 terms in the representation (13) are nonzero (three for each possible orientation of $f(z)$ ). Thus, the lemma will follow once we establish that

$$
c_{f}(z) \leq 2^{k}, \quad \text { for all } f \in \mathcal{F}_{k} .
$$

This will be done by induction on $k$. For $k=1$, the statement is obvious. Suppose (14) holds for $k=m$. Fix $f \in \mathcal{F}_{m+1}$, say $f(z)= \pm 2^{m+1} z+j$. If $j$ is even, then every $\mathbf{i} \in I_{m+1}$ with $f_{\mathbf{i}}=f$ must have $i_{m+1} \in\{1,2\}$. Thus, $\operatorname{card}(\mathcal{P}(f)) \leq 2$, and by the induction hypothesis,

$$
c_{f}(z)=\sum_{\hat{f} \in \mathcal{P}(f)} c_{\hat{f}}(z) \leq 2 \cdot 2^{m}=2^{m+1} .
$$

If $j$ is odd, then each $\mathbf{i} \in I_{m+1}$ with $f_{\mathbf{i}}=f$ must have $i_{m+1}=0$, and $f$ has a unique predecessor $\hat{f} \in \mathcal{F}_{m}$. The induction hypothesis gives

$$
c_{f}(z)=2 c_{\hat{f}}(z) \leq 2^{m+1} .
$$

Thus, (14) holds for all $k \in \mathbf{N}$, and the lemma follows.

Lemma $3.4 \mathcal{H}^{3 / 2}(\hat{Z})>0$.

Proof. Let $\lambda$ denote Lebesgue measure on $[0,1]$. We define a measure $\mu$ on the Borel sets of $[0,1] \times \mathbf{R}$ by

$$
\mu(U):=\lambda(\{t: U \cap \hat{Z} \cap(\{t\} \times \mathbf{R}) \neq \emptyset\}) .
$$

Thus, $\mu$ is a probability measure concentrated on $\hat{Z}$, whose projection onto the horizontal axis is $\lambda$. We will show that there exists a positive constant $C$ such that for every square $U$ with sufficiently small diameter,

$$
\mu(U) \leq C|U|^{3 / 2} .
$$


It will then follow that for every countable cover $\left\{U_{i}\right\}$ of $\hat{Z}$ consisting of sets with sufficiently small diameter,

$$
1 \leq \mu\left(\bigcup_{i} U_{i}\right) \leq C \sum_{i}\left|U_{i}\right|^{3 / 2},
$$

so $\sum_{i}\left|U_{i}\right|^{3 / 2} \geq C^{-1}$, and hence $\mathcal{H}^{3 / 2}(\hat{Z}) \geq C^{-1}>0$.

Fix a square $U$ with sides parallel to the coordinate axes, say $U=\left[t_{0}, t_{0}+\right.$ $s] \times\left[z_{0}, z_{0}+s\right]$. Let $n$ be the unique integer such that $4^{-n} \leq s<4^{-(n-1)}$. Thus, $\left[t_{0}, t_{0}+s\right]$ intersects at most 4 of the intervals $\left[i / 4^{n},(i+1) / 4^{n}\right]$.

Similarly, we may assume $s$ is small enough (and $n$ is large enough) so that the interval $\left[z_{0}, z_{0}+s\right]$ intersects at most two of the intervals $\left[j / 2^{n+1},(j+\right.$ $\left.1) / 2^{n+1}\right]$, with $j \in \mathbf{Z}$. This will certainly be the case if $s<2^{-(n+1)}$, and sufficient for this is $4^{-(n-1)} \leq 2^{-(n+1)}$, which holds for $n \geq 3$. In the same way, $\left[z_{0}, z_{0}+s\right]$ intersects at most two of the intervals $\left[j / 2^{n+r+1},(j+1) / 2^{n+r+1}\right]$ for $r=1,2, \ldots, n-3$.

Now fix one of the intervals $\left[i / 4^{n},(i+1) / 4^{n}\right]$ that intersect $\left[t_{0}, t_{0}+s\right]$, and denote the chosen interval by $I$. Let $V:=U \cap(I \times \mathbf{R})$. Since the intersection of the vertical strip $I \times \mathbf{R}$ with $\hat{Z}$ is an affine-contracted copy of $\hat{Z}$, the number of stage- $(n+r)$ rectangles intersected by the line segment $I \times\{z\}$ is constant in $z$ on each interval $\left(j / 2^{n+r+1},(j+1) / 2^{n+r+1}\right)$, and by Lemma 3.3 , is at most $6 \cdot 2^{r}$. Thus, by the last statement in the previous paragraph, the number of stage- $(n+r)$ rectangles that intersect $V$ is at most $12 \cdot 2^{r}$, for $r=1,2, \ldots, n-3$. Since each such rectangle has width $4^{-(n+r)}$, it follows that

$$
\mu(V) \leq 12 \cdot 2^{r} \cdot 4^{-(n+r)}=12 \cdot 2^{-(2 n+r)}, \quad r=1,2, \ldots, n-3,
$$

and thus,

$$
\mu(V) \leq 12 \cdot 2^{-(3 n-3)} .
$$

Repeating the above argument for each of the (at most 4$)$ intervals $\left[i / 4^{n},(i+\right.$ 1) $\left./ 4^{n}\right]$ that intersect $\left[t_{0}, t_{0}+s\right]$, we obtain that

$$
\begin{aligned}
\mu(U) & \leq 4 \cdot 12 \cdot 2^{-(3 n-3)}=3 \cdot 2^{7} \cdot 2^{-3 / 4}\left(4^{-n} \sqrt{2}\right)^{3 / 2} \\
& \leq 3 \cdot 2^{7} \cdot 2^{-3 / 4}|U|^{3 / 2},
\end{aligned}
$$

as desired.

Note that the proofs of Lemmas 3.2 and 3.4 yield the estimates

$$
.00219 \approx\left(3 \cdot 2^{7} \cdot 2^{-3 / 4}\right)^{-1} \leq \mathcal{H}^{3 / 2}(\hat{Z}) \leq 5 \cdot 2^{-1 / 4} \approx 4.204 .
$$

While these bounds can be improved, it seems difficult to determine the exact value of $\mathcal{H}^{3 / 2}(\hat{Z})$.

Proof of Theorem 3.1. Immediate from Lemmas 3.2 and 3.4, and the relationship between $X, Y$, and $\hat{Z}$. 


\section{Acknowledgement}

The second author wishes to thank the Department of Mathematics of the University of North Texas for its continued support.

\section{References}

[1] S. Bailey, T. Kim and R. Strichartz, Inside the Lévy dragon, Amer. Math. Monthly 109, 689-703 (2002).

[2] G. DE Rham, Sur quelques courbes définies par des équations fonctionnelles, Rend. Sem. Mat. Torino 16, 101-113 (1957).

[3] P. Duval and J. Keesling, The Hausdorff dimension of the boundary of the Lévy dragon, Int. J. Math. and Math. Sci. 20, 627-632 (1997).

[4] G. A. EdGar, Classics on Fractals, Addison-Wesley, Reading, MA (1993).

[5] G. A. Edgar, Kiesswetter's fractal has Hausdorff dimension 3/2, Real Anal. Exchange 14, 215-223 (1988/89).

[6] K. J. FALCONeR, The geometry of fractal sets. Cambridge University Press (1985).

[7] K. J. Falconer, The Hausdorff dimension of self-affine fractals. Math. Proc. Camb. Phil. Soc. 103, no.2, 339-350 (1988).

[8] K. J. Falconer, The dimension of self-affine fractals II. Math. Proc. Camb. Phil. Soc. 111, no.1, 169-179 (1992).

[9] J. E. Hutchinson, Fractals and self-similarity. Indiana Univ. Math. J. 30, no. 5, 713-747 (1981).

[10] T. Kamae, A characterization of self-affine functions, Japan J. Appl. Math. 3, 271-280 (1986).

[11] R. Kenyon and Y. Peres, Hausdorff dimensions of sofic affine-invariant sets, Israel J. Math. 94, 157-178 (1996).

[12] N. Kono, On self-affine functions, Japan J. Appl. Math. 3, 259-269 (1986).

[13] P. LÉvy, Les courbes planes ou gauches et les surfaces composées de parties semblables au tout, J. Ecole Polytechn., 227-247, 249-291 (1938).

[14] R. D. Mauldin and S. C. Williams, Hausdorff dimension in graph directed constructions. Trans. Amer. Math. Soc. 309, no. 2, 811-829 (1988).

[15] M. MCCLuRE, The Hausdorff dimension of Hilbert's coordinate functions, Real Anal. Exchange 24, 875-884 (1998/9).

[16] H. SAGan, Space-Filling Curves, Springer-Verlag, New York, (1994). 
[17] S. Takahashi, Dimension spectra of self-affine sets, Israel J. Math. 127, 1-18 (2002). 